\title{
Initial results of pulmonary resection after neoadjuvant nivolumab in patients with resectable non-small cell lung cancer
}

\author{
Matthew J. Bott, MD, ${ }^{a}$ Stephen C. Yang, MD, ${ }^{\mathrm{b}}$ Bernard J. Park, MD, ${ }^{\mathrm{a}}$ Prasad S. Adusumilli, MD, ${ }^{\mathrm{a}}$ \\ Valerie W. Rusch, MD, ${ }^{\mathrm{a}}$ James M. Isbell, MD, ${ }^{\mathrm{a}}$ Robert J. Downey, MD, ${ }^{\mathrm{a}}$ Julie R. Brahmer, MD, \\ Richard Battafarano, MD, PhD, ${ }^{\mathrm{b}}$ Errol Bush, MD, ${ }^{\mathrm{b}}$ Jamie Chaft, MD, ${ }^{\mathrm{d}}$ Patrick M. Forde, MD, \\ David R. Jones, MD, ${ }^{a}$ and Stephen R. Broderick, MD, MPHS ${ }^{b}$
}

\section{ABSTRACT}

Objective: We conducted a phase I trial of neoadjuvant nivolumab, a monoclonal antibody to the programmed cell death protein 1 checkpoint receptor, in patients with resectable non-small cell lung cancer. We analyzed perioperative outcomes to assess the safety of this strategy.

Methods: Patients with untreated stage I-IIIA non-small cell lung cancer underwent neoadjuvant therapy with 2 cycles of nivolumab $(3 \mathrm{mg} / \mathrm{kg}), 4$ and 2 weeks before resection. Patients underwent invasive mediastinal staging as indicated and post-treatment computed tomography. Primary study end points were safety and feasibility of neoadjuvant nivolumab followed by pulmonary resection. Data on additional surgical details were collected through chart review.

Results: Of 22 patients enrolled, 20 underwent resection. One was unresectable; another had small cell histologic subtype. There were no delays to surgical resection. Median time from first treatment to surgery was 33 (range, 17-43) days. There were 15 lobectomies, 2 pneumonectomies, 1 bilobectomy, 1 sleeve lobectomy, and 1 wedge resection. Of 13 procedures attempted via a videoassisted thoracoscopic surgery or robotic approach, 7 (54\%) required thoracotomy. Median operative time was 228 (range, 132-312) minutes; estimated blood loss was 100 (range, 25-1000) $\mathrm{mL}$; length of hospital stay was 4 (range, 2-17) days. There was no operative mortality. Morbidity occurred in 10 of 20 patients $(50 \%)$. The most common postoperative complication was atrial arrhythmia $(6 / 20 ; 30 \%)$. Major pathologic response was identified in 9 of 20 patients (45\%).

Conclusions: Neoadjuvant therapy with nivolumab was not associated with unexpected perioperative morbidity or mortality. More than half of the video-assisted thoracoscopic surgery/robotic cases were converted to thoracotomy, often because of hilar inflammation and fibrosis. (J Thorac Cardiovasc Surg 2019;158:269-76)

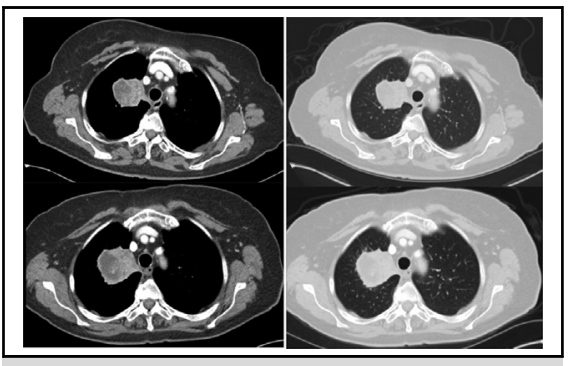

Before (top) and after (bottom) nivolumab treatment. Imaging often underestimates pathologic response.

\section{Central Message}

Pulmonary resection after neoadjuvant therapy with nivolumab did not result in undue morbidity or mortality. Major pathologic responses were identified despite stable disease radiographically.

\section{Perspective}

Immune checkpoint inhibitors have durable efficacy in advanced NSCLC, and their role in the neoadjuvant setting is under investigation. Pulmonary resection after neoadjuvant programmed cell death protein 1 blockade can be done without excessive treatmentrelated toxicity, operative morbidity, or mortality. Thoracic surgeons should play an active role in the planning and execution of clinical trials in this developing field.

See Commentary on page 277 .
Immune checkpoint inhibitors have rapidly become a pivotal treatment option for patients with advanced non-small cell lung cancer (NSCLC). These agents, which block immune

\footnotetext{
From the ${ }^{\text {a}}$ Thoracic Service, Department of Surgery, Memorial Sloan Kettering Cancer Center, New York, NY; ${ }^{b}$ Division of Thoracic Surgery, Department of Surgery, ${ }^{\mathrm{c}}$ Sidney Kimmel Cancer Center, The Johns Hopkins Medical Institutions, Baltimore, Md; and ${ }^{\mathrm{d}}$ Department of Medical Oncology, Memorial Sloan-Kettering Cancer Center, and Weill Cornell Medical College, New York, NY.

This study was supported, in part, by National Institutes of Health Cancer Center Support Grant P30 CA008748.

Read at the 98th Annual Meeting of The American Association for Thoracic Surgery, San Diego, California, April 28-May 1, 2018.
}

inhibitory signals on the cancer cell surface, allow for recognition of tumor antigens by the patient's $T$ lymphocytes and ultimately result in destruction of tumor cells by the immune

\footnotetext{
Received for publication April 30, 2018; revisions received Oct 18, 2018; accepted for publication Nov 2, 2018; available ahead of print Feb 1, 2019

Address for reprints: Stephen R. Broderick, MD, MPHS, 600 N Wolfe St, Blalock 240, Baltimore, MD 21287 (E-mail: sbroder7@jhmi.edu). 0022-5223/\$36.00

Copyright (C) 2018 by The American Association for Thoracic Surgery https://doi.org/10.1016/j.jtcvs.2018.11.124
} 


\section{Abbreviations and Acronyms}

$\mathrm{CT}=$ computed tomography

MPR = major pathologic response

NSCLC $=$ non-small cell lung cancer

PD-L1 = programmed death-ligand 1

To view the AATS Annual Meeting

Webcast, see the URL next to the

webcast thumbnail.

system. Initial studies of these drugs in patients with metastatic NSCLC and melanoma suggested that they are capable of producing significant and often durable treatment responses in subsets of patients with metastatic cancer. ${ }^{1,2}$ Long-term outcomes from clinical trials of nivolumab in previously treated patients with advanced NSCLC showed a 2-year overall survival of $23 \%$ to $29 \%$ and 5 -year overall survival of $16 \%{ }^{1,3}$ Subsequent studies using these agents in combination with chemotherapy and radiation also suggested promising efficacy in patients with locally advanced NSCLC. ${ }^{4}$ Recent results of the PACIFIC trial showed a 12-month progression-free survival of 16.8 months in patients with unresectable stage III NSCLC treated with definitive chemoradiotherapy followed by durvalumab (anti-programmed death-ligand 1 [PD-L1]) versus 5.6 months in patients given placebo. ${ }^{4}$ The success of these agents in the metastatic setting has generated considerable enthusiasm among clinicians, and high-visibility marketing campaigns have resulted in rapidly expanding interest among patients.

On the basis of the success of these trials, numerous studies have been initiated to evaluate the utility of immunotherapeutic agents as neoadjuvant or adjuvant therapy in patients with surgically resectable NSCLC. The administration of these agents in the preoperative setting offers several theoretical advantages. Drug treatment while the tumor is in situ might provide immunologic benefits in terms of increased antigen load and might facilitate tumor recognition and drug efficacy. ${ }^{5}$ Similarly, correlative studies on post-treatment tumor specimens offer a unique opportunity to study underlying biologic and immunologic mechanisms and could yield important insights regarding treatment sensitivity and resistance. Despite these potential benefits, data regarding the safety and feasibility of surgical resection after treatment in this setting remain scarce. Initial retrospective case series in patients with previously unresectable or metastatic disease have suggested overall feasibility but have also raised concerns about technical challenges and perioperative issues unique to this patient population. 6,7
We recently completed a clinical trial on the safety and feasibility of the anti-programmed cell death protein 1 antibody nivolumab as a neoadjuvant treatment in patients with surgically resectable NSCLC. Data regarding tolerability and efficacy of this treatment approach have been recently reported. ${ }^{8}$ However, the unique mechanism of action of these agents and the theoretical challenges this might pose for subsequent lung resection warrants a more granular analysis of operative details and postoperative outcomes in this patient cohort. The aim of this study was to further explore the implications of preoperative nivolumab treatment on subsequent lung resection in these patients, with particular attention given to technical details and postoperative outcomes.

\section{METHODS}

The study was approved by the institutional review boards at the 2 participating study sites, Johns Hopkins Hospital and Memorial Sloan Kettering Cancer Center (MSK IRB \#15-106, August 21, 2015; JHMI protocol NA_00092076/ J1414), and all patients gave written informed consent. The clinical trial was registered at www.clinicaltrials.gov (NCT02259621). The trial details were previously published ${ }^{8}$ but are summarized in brief herein. The trial was a single-arm study that involved 2 doses of nivolumab $(3 \mathrm{mg} / \mathrm{kg}$ ) administered intravenously 4 weeks and 2 weeks before surgery. Entry criteria included age 18 years or older with stage I to IIIA biopsy-proven NSCLC. Eligible patients were evaluated by a thoracic surgeon before enrollment, to confirm resectability. Nivolumab was the sole neoadjuvant therapy; patients were eligible for adjuvant therapy with chemotherapy or radiation as indicated. Patients were specifically excluded if they had a history of immunodeficiency, had active autoimmune or infectious disease, were receiving immunosuppressive therapy, or had a concurrent malignancy. All patients underwent a standard preoperative staging workup that included pretreatment tumor biopsy, contrast-enhanced computed tomography (CT) scan of the chest, positron emission tomography/CT scan, and invasive mediastinal nodal staging with endobronchial ultrasound or mediastinoscopy as indicated. Patients also underwent brain imaging with CT or magnetic resonance imaging when clinically indicated and post-treatment CT before resection. Resection of the primary tumor and lymphadenectomy were performed according to institutional standards.

Patient demographic characteristics, and pathologic and clinical outcomes data were prospectively collected in accordance with the study protocol. To collect additional operative details, we performed a retrospective chart review on the study patients. Retrospectively abstracted data include operative approach, extent of resection, operative time, hospital length of stay, postoperative morbidity, rationale for conversion of minimally invasive surgical procedures to thoracotomy if necessary, and other details related to the operative experience.

Tumors were staged according the seventh edition of the American Joint Committee on Cancer Tumor, Node, Metastases staging criteria. Major pathologic response was defined as $\leq 10 \%$ of viable tumor cells remaining on postoperative pathologic review. ${ }^{9}$ Operative time was extracted from the operating room documentation and was defined as the duration from incision to wound closure. Surgical complications were defined according to the Society of Thoracic Surgeons database criteria.

\section{RESULTS}

Between August 2015 and October 2016, 22 patients were enrolled in the study and received neoadjuvant treatment with nivolumab. One patient was subsequently 
removed when histologic analysis of a preoperative biopsy showed small cell carcinoma. Histologic subtypes for the remaining patients were adenocarcinoma (14/21 [67\%]), squamous cell carcinoma (5/21 [24\%]), pleomorphic carcinoma (1/21 [5\%]), and adenosquamous carcinoma $(1 / 21[5 \%])$. Preoperative clinical staging was as follows: 4 of $21(19 \%)$ stage I, 10 of $21(48 \%)$ stage II, and 7 of $21(33 \%)$ stage IIIA. Median age was 67 years (range, 55-84 years). Eleven patients (52\%) were female; and 18 $(86 \%)$ were current or former smokers (Table 1). Twenty patients underwent resection. A single patient with a T2N2 squamous cell tumor developed a postobstructive pneumonia after a single dose of nivolumab and underwent uncomplicated resection at that time; this was the only grade $\geq 3$ adverse effect of nivolumab treatment. One patient with stage IIIA disease was deemed unresectable because of tracheal invasion noted on preoperative bronchoscopy, and surgery was aborted. Most patients $(18 / 21$ [86\%]) had stable disease on post-treatment CT imaging. Two patients $(10 \%)$ had a partial response, and $1(5 \%)$ had radiographic progression of disease. There were no treatment-related delays to scheduled resections. Median time from the second dose of nivolumab to resection was 18 days (range, 11-29 days).

Of the 20 patients who underwent resection, 15 (75\%) underwent lobectomy, $2(10 \%)$ underwent pneumonectomy, 1 $(5 \%)$ each underwent sleeve lobectomy, bilobectomy, and wedge resection. Two of the lobectomies included en bloc wedge resection from an adjoining lobe. Fourteen of the 20 resections $(70 \%)$ were performed via an open approach, of which 7 cases $(35 \%)$ used thoracotomy as the initial strategy. Of the 13 cases that were initiated using a minimally invasive (video-assisted thoracoscopic surgery or robotic) approach, $7(54 \%)$ were converted to thoracotomy.

Conversion to thoracotomy was performed in 1 of 4 $(25 \%)$ patients with clinical stage I tumors. Operative reports note that this was because of dense, vascularized chest wall adhesions. Similarly, 1 of 2 clinical stage IIA cases were converted to thoracotomy. This patient had a postobstructive pneumonia preoperatively, and conversion was required because of dense adhesions in the fissure and between the left lower lobe and the aorta. The remainder of conversions occurred in patients with more advanced stages of disease (5 of 7 [71\%] stage IIB and IIIA). Also, 5 of 8 cases $(62 \%)$ with either clinical or pathologic evidence of lymph node metastases that were attempted thoracoscopically required conversion. Operative reports from these cases note inflammation and/or dense adhesions in either the fissure or surrounding hilar and mediastinal nodal stations, which contributed to conversion. The presence of a major pathologic response (MPR) did not appear to be a factor in conversion to an open approach, because 4 of 6 cases with MPR (67\%)
TABLE 1. Characteristics of enrolled patients $(\mathrm{N}=21)$

\begin{tabular}{|c|c|}
\hline Characteristic & Value \\
\hline Median age (range), y & $67(55-84)$ \\
\hline \multicolumn{2}{|l|}{ Sex } \\
\hline Female & $11(52)$ \\
\hline Male & $10(48)$ \\
\hline \multicolumn{2}{|l|}{ Clinical stage } \\
\hline IA T1N0 & $2(10)$ \\
\hline IB T2NO & $2(10)$ \\
\hline IIA & $5(24)$ \\
\hline T1bN1 & $1(5)$ \\
\hline T2aN1 & $4(19)$ \\
\hline IIB & $5(24)$ \\
\hline T3N0 & $3(14)$ \\
\hline $\mathrm{T} 2 \mathrm{bN} 1$ & $2(10)$ \\
\hline IIIA & $7(33)$ \\
\hline $\mathrm{T} 1 \mathrm{~N} 2$ & $2(10)$ \\
\hline $\mathrm{T} 2 \mathrm{~N} 2$ & $2(10)$ \\
\hline $\mathrm{T} 3 \mathrm{~N} 1$ & $2(10)$ \\
\hline $\mathrm{T} 4 \mathrm{~N} 1$ & $1(5)$ \\
\hline \multicolumn{2}{|l|}{ Histologic subtype } \\
\hline Adenocarcinoma & $14(67)$ \\
\hline Squamous cell & $5(24)$ \\
\hline Adenosquamous & $1(5)$ \\
\hline Pleomorphic & $1(5)$ \\
\hline \multicolumn{2}{|l|}{ Smoking history } \\
\hline Never & $3(14)$ \\
\hline Former/current & $18(86)$ \\
\hline \multicolumn{2}{|c|}{ Extent of resection $(n=20)$} \\
\hline Lobectomy & $15(75)$ \\
\hline Pneumonectomy & $2(10)$ \\
\hline Wedge & $1(5)$ \\
\hline Sleeve lobectomy & $1(5)$ \\
\hline Bilobectomy & $1(5)$ \\
\hline \multicolumn{2}{|l|}{ Approach $(\mathrm{n}=20)$} \\
\hline Thoracotomy & $14(70)$ \\
\hline Thoracoscopy & $3(14)$ \\
\hline Robotic & $3(14)$ \\
\hline
\end{tabular}

Unless otherwise indicated, data are $\mathrm{n}(\%)$.

that were attempted thoracoscopically were completed in this manner.

Perioperative outcome data are summarized in Table 2. Median surgical time was 228 minutes (range, 132-312 minutes). Median estimated blood loss was $100 \mathrm{~mL}$ (range, 25-1000 mL). No patient required blood transfusion. Median hospital length of stay was 4 days (range, 2-17 days). There was no operative mortality. A single patient died of traumatic injury on postoperative day 61 . Ten of 20 patients $(50 \%)$ experienced any postoperative morbidity. Atrial arrhythmia was the most common morbidity, occurring in 6 patients $(30 \%)$. One of these patients suffered a concomitant myocardial infarction. A single patient developed pneumonia and a pulmonary embolism. Prolonged air leak and urinary retention each 
TABLE 2. Perioperative outcomes of 20 patients who underwent pulmonary resection after neoadjuvant nivolumab treatment

\begin{tabular}{lc}
\hline \multicolumn{1}{c}{ Perioperative outcome } & Value \\
\hline Median surgical time (range), min & $228(132-312)$ \\
\hline Median estimated blood loss (range), mL & $100(25-1000)$ \\
\hline Median length of stay (range), d & $4(2-17)$ \\
Operative mortality & $0^{*}$ \\
\hline Any morbidity & $10(50)$ \\
Atrial arrhythmia & $6(30)$ \\
Pneumonia & $1(5)$ \\
Pulmonary embolism & $1(5)$ \\
Myocardial infarction & $1(5)$ \\
Prolonged air leak & $1(5)$ \\
Urinary retention & $1(5)$ \\
Empyema & $1(5)$ \\
\hline
\end{tabular}

*One patient died of traumatic injury on postoperative day 61 .

occurred in a single patient. A single patient who underwent lobectomy developed an empyema 4 months postoperatively requiring flap drainage. A single patient who underwent thoracoscopic lobectomy experienced an injury to the bronchus intermedius during dissection of the subcarinal space requiring conversion to thoracotomy for repair.

On pathologic analysis, 9 of 20 patients $(45 \%)$ had an MPR (defined as $\leq 10 \%$ residual viable tumor) ${ }^{8,9} ; 8$ $(40 \%)$ had pathologic downstaging. Two patients (pretreatment $\mathrm{T} 1 \mathrm{bN} 1$ pleomorphic carcinoma and $\mathrm{T} 3 \mathrm{~N} 1$ squamous) had no residual viable tumor on final pathologic assessment. Currently, median follow-up is 20 months, with 18 of 20 patients $(90 \%)$ alive at last follow-up. Three of 20 patients $(15 \%)$ developed recurrent disease: 1 patient with solitary brain metastasis 2 months postresection treated with stereotactic radiotherapy, 1 patient with mediastinal nodal recurrence treated with definitive chemoradiation, and 1 patient with distant metastatic disease. One resected patient has died of NSCLC.

\section{DISCUSSION}

Several prospective randomized trials have shown improved survival and tolerability with programmed cell death protein 1 and PD-L1 blockade in select patients with metastatic NSCLC, in combination with platinumdoublet chemotherapy and as a single agent, compared with chemotherapy. ${ }^{10-13}$ The improved disease-free and overall survival at 24 months with immunotherapy, compared with chemotherapy, in the metastatic setting has led to multiple clinical trials of immunotherapeutic agents in the neoadjuvant setting (Table 3). Evaluation of perioperative outcomes from these trials is necessary to address the feasibility and safety of pulmonary resection after neoadjuvant immunotherapy. These surgical outcomes are relevant to the planning and execution of future trials and surgical practice.

Chaft and colleagues first reported a series of 5 patients with advanced NSCLC who underwent pulmonary resection for persistent local disease after treatment with T-cell checkpoint inhibitors. ${ }^{7}$ This report suggested that pulmonary resection was feasible but cautioned that mediastinal and hilar fibrosis might develop as a result of response to treatment. More recently, Bott and colleagues reported a series of 19 patients who underwent pulmonary resection for metastatic disease or previously unresectable NSCLC and concluded that these resections, although challenging, were feasible, without undue morbidity. ${ }^{6}$ Finally, Yang and colleagues reported surgical outcomes from TOP1201, a phase II trial that evaluated the feasibility and safety of neoadjuvant chemotherapy with ipilimumab (monoclonal antibody to cytotoxic T-lymphocyte-associated protein 4) in patients with stage II/III NSCLC. ${ }^{14}$ Similarly, this study showed that resection in these patients was safe and feasible, with perioperative outcomes similar to those in a cohort of patients who received neoadjuvant platinumbased chemotherapy.

The current study represents the largest experience to date of planned pulmonary resection after the neoadjuvant administration of immunotherapeutic agents in resectable NSCLC. Pulmonary resection in 20 patients with stage I to IIIA NSCLC after neoadjuvant treatment with 2 doses of nivolumab did not result in unexpected morbidity or mortality. There were no operative mortalities-a single patient died of a traumatic injury on postoperative day 61 . This compares favorably with the $0 \%$ to $7 \%$ mortality in previous trials of neoadjuvant chemotherapy followed by pulmonary resection. ${ }^{15-20}$ In addition, rates of perioperative morbidities were similar to those reported in previous multicenter trials. ${ }^{15-20}$ Specifically, compared with the Southwest Oncology Group (SWOG) S9900 trial, which compared chemotherapy followed by surgery with surgery alone in patients with stage IB to IIIA NSCLC, patients in the current study showed favorable rates of pneumonia $(5 \%$ vs $7 \%)$, reintubation $(0 \%$ vs $7 \%$ ), prolonged air leak ( $5 \%$ vs $9 \%$ ), and respiratory failure $(0 \%$ vs $7 \%)$. The rate of atrial arrhythmia was higher in this study than in SWOG S9900 $(30 \%$ vs $16 \%){ }^{15}$ It is unclear whether this is because of the treatment strategy or instead is related to the relatively small size of the study.

Most resections (14/20) in this study were performed via an open approach. In 7 of 20 cases, this was the initial approach selected by the operating surgeon. The remainder were cases that were converted from an initial minimally invasive approach. Because several of these cases involved sizeable tumors with hilar and/or mediastinal nodal disease, the necessity of conversion is not surprising. However, in 
TABLE 3. Select ongoing trials of neoadjuvant immunotherapeutic agents in patients with resectable non-small cell lung cancer

\begin{tabular}{lllllr}
\hline Trial identifier & Phase & Stage & \multicolumn{1}{c}{ Neoadjuvant intervention } & Primary end point & Target accrual \\
\hline NCT02998528 & III & IB-IIIA & $\begin{array}{l}\text { Nivolumab with ipilimumab vs nivolumab } \\
\text { with platinum doublet vs platinum doublet }\end{array}$ & MPR & 624 \\
NCT03158129 & II & I-IIIA & Nivolumab with or without ipilimumab & MPR & 66 \\
NCT02259621 & II & IB-IIIA & Nivolumab with or without ipilimumab & MPR & 30 \\
NCT02927301 & II & IB-IIIA & Atezolizumab & MPR & Event-free survival \\
NCT02572843 & II & IIIA(N2) & Cisplatin/docetaxel with durvalumab & 68 \\
NCT02818920 & II & IB-IIIA & Pembrolizumab (neoadjuvant and adjuvant) & Surgical feasibility \\
NCT03237377 & II & IIIA & Durvalumab with radiation & Safety & 32 \\
\hline
\end{tabular}

$M P R$, Major pathologic response.

most cases, this appeared to be related to inflammatory response and fibrosis at the primary tumor and involved nodal stations, presumably related to treatment response. Conversion to thoracotomy appeared to be more frequent in more advanced stages of disease $(25 \%$ of stage I cases, compared with $71 \%$ of stage IIB/IIIA cases). Furthermore, $62 \%$ of patients with node-positive tumors (either clinical or pathologic) who underwent an attempt at minimally invasive resection were converted to thoracotomy. Interestingly, the degree of pathologic response to treatment did not seem to be a significant factor, because most of the MPR cases $(67 \%)$ that were attempted thoracoscopically were completed without conversion.

Whereas some studies have suggested that minimally invasive lobectomy after neoadjuvant chemotherapy is feasible in patients with stage IIIA (N2) disease, ${ }^{21-23}$ a recent analysis of the National Cancer Database by Krantz and colleagues showed that lobectomy was accomplished via a minimally invasive approach in only $25.7 \%$ of such patients. $^{24}$ In the study by Bott and colleagues, 4 of 11 anatomic resections $(36 \%)$ were accomplished via a minimally invasive approach. ${ }^{6}$ The surgical approach was not reported in the operative results of the TOP1201 trial. $^{14}$ Although the current study included few patients, the necessity of thoracotomy does not seem to have affected morbidity and early mortality rates.

It is evident from this study that radiologic assessment of treatment response with $\mathrm{CT}$ might not be accurate after neoadjuvant immunotherapy. This is an important consideration as trials continue to accrue and for clinical practice. Major pathologic response was identified in 9 of 20 of patients $(45 \%)$, whereas post-treatment CT most commonly showed stable disease, with partial response in only 2 patients and progression in 1 patient. The 2 patients in the current study with complete pathologic response radiographically showed stable disease. This phenomenon, oftentimes referred to as "pseudo-progression,",25,26 has been previously documented and is likely related to T-cell infiltration and peritumoral inflammation during the early stages of treatment.
In addition to this study, 2 currently under way trials are evaluating combination nivolumab and ipilimumab in the neoadjuvant setting. CheckMate 816 (NCT02998528) is a multicenter randomized trial that is comparing neoadjuvant nivolumab/ipilimumab combination with nivolumab and platinum doublet or platinum doublet alone in patients with stage IB $(>4 \mathrm{~cm})$ to IIIA resectable NSCLC. The NEOSTAR trial (NCT03158129) is comparing the efficacy of the nivolumab/ipilimumab combination with nivolumab monotherapy in the neoadjuvant setting in patients with stage I to IIIA NSCLC. The rationale for the additional use of ipilimumab is on the basis of results from the CheckMate 012 study, which showed improved progression-free survival, compared with nivolumab monotherapy, in patients with stage III/IV NSCLC. ${ }^{27,28}$ Additional neoadjuvant trials are under way of other immunotherapeutic agents, including the PDL1 antibody atezolizumab in a multi-institutional trial through the Lung Cancer Mutation Consortium (NCT02927301), durvalumab (NCT02572843), and pembrolizumab alone or in combination with chemotherapy (Table 3).

\section{CONCLUSIONS}

As the use of T-cell checkpoint inhibitors in the treatment of NSCLC becomes more widespread in clinical practice and as data from trials of these drugs administered in the neoadjuvant setting accrue, it is likely that surgeons will be asked to perform pulmonary resections on increasing numbers of patients who have received immunotherapy. It is incumbent on surgeons to report surgical outcomes under this novel treatment strategy. In this analysis we report the perioperative outcomes in, to our knowledge, the largest series to date of patients treated with neoadjuvant nivolumab for resectable NSCLC. Although pulmonary resection in this setting might be challenging, our experience suggests that these procedures can be accomplished safely with outcomes similar to historical outcomes after neoadjuvant chemotherapy. The relative effectiveness of this strategy compared with neoadjuvant platinum-based chemotherapy will hopefully be clarified by currently accruing clinical trials. 


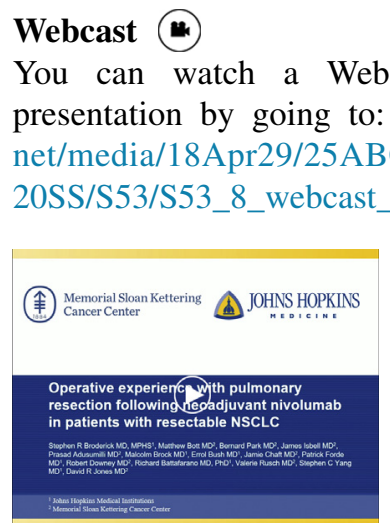

\section{Conflict of Interest Statement}

M.J.B. is a consultant for AstraZeneca Pharmaceuticals. S.R.B. is a consultant for Bristol-Meyers-Squibb. J.R.B. is a consultant for Celgene, Lilly, Merck, and Bristol-Meyers-Squibb; she receives grant funding from Bristol-Meyers-Squibb and MedImmune/AstraZeneca. J.C. is a consultant for Merck, Bristol-Meyers-Squibb, Genentech, and AstraZeneca; she receives grant funding from Stand Up To Cancer and Memorial Sloan-Kettering Cancer Center. P.M.F. is a consultant for AstraZeneca, Bristol-Meyers-Squibb, Merck, Novartis, Kyowa, Inivata, EMD Serono, and Boehringer Ingelheim; he receives grant funding from Bristol-Meyers-Squibb, LUNGevity, IASLC, ECOG-ACRIN Foundation, and Stand Up To Cancer. V.W.R. receives grant support from Stand Up To Cancer, Memorial Sloan-Kettering Cancer Center, and Genelux. All other authors have nothing to disclose with regard to commercial support.

\section{References}

1. Horn L, Spigel DR, Vokes EE, Holgado E, Ready N, Steins M, et al. Nivolumab versus docetaxel in previously treated patients with advanced non-small-cell lung cancer: two-year outcomes from two randomized, open-label, phase III trials (Checkmate 017 and Checkmate 057). J Clin Oncol. 2017;35:3924-33.

2. Callahan MK, Kluger H, Postow MA, Segal NH, Lesokhin A, Atkins MB, et al. Nivolumab plus ipilimumab in patients with advanced melanoma: updated survival, response, and safety data in a phase I dose-escalation study. J Clin Oncol. 2018;36:391-8.

3. Gettinger S, Horn L, Jackman D, Spigel D, Antonia S, Hellmann M, et al. Five-year follow up of nivolumab in previously treated advanced non-smallcell lung cancer: results from the CA209-003 study. J Clin Oncol. 2018;36: $1675-84$.

4. Antonia SJ, Villegas A, Daniel D, Vicente D, Murakami S, Hui R, et al. Durvalumab after chemoradiotherapy in stage III non-small-cell lung cancer. N Engl J Med. 2017;377:1919-29.

5. Yi JS, Ready N, Healy P, Dumbauld C, Osborne R, Berry M, et al. Immune activation in early-stage non-small-cell lung cancer patients receiving neoadjuvant chemotherapy plus ipilimumab. Clin Cancer Res. 2017;23:7474-82.

6. Bott MJ, Cools-Lartigue J, Tan KS, Dycoco J, Bains MS, Downey RJ, et al. Safety and feasibility of lung resection after immunotherapy for metastatic or unresectable tumors. Ann Thorac Surg. 2018;106:178-83.

7. Chaft JE, Hellmann MD, Velez MJ, Travis WD, Rusch VW. Initial experience with lung cancer resection after treatment with T-cell checkpoint inhibitors. Ann Thorac Surg. 2017;104:e217-8.
8. Forde PM, Chaft JE, Smith KM, Anagnostou V, Cottrell TR, Hellmann MD, et al. Neoadjuvant PD-1 blockade in resectable lung cancer. N Engl J Med. 2018;378: 1976-86.

9. Pataer A, Kalhor N, Correa AM, Raso MG, Erasmus JJ, Kim ES, et al. Histopathologic response criteria predict survival of patients with resected lung cancer after neoadjuvant chemotherapy. J Thorac Oncol. 2012;7:825-32.

10. Borghaei H, Paz-Ares L, Horn L, Spigel DR, Steins M, Ready NE, et al. Nivolumab versus docetaxel in advanced nonsquamous non-small-cell lung cancer. N Engl J Med. 2015;373:1627-39.

11. Fehrenbacher L, Spira A, Ballinger M, Kowanetz M, Vansteenkiste J, Mazieres J, et al. Atezolizumab versus docetaxel for patients with previously treated non-small-cell lung cancer (POPLAR): a multicenter, open-label, phase 2 randomised controlled trial. Lancet. 2016;387:1837-46.

12. Garon EB, Rizvi NA, Hui R, Leighl N, Balmanoukian AS, Eder JP, et al. Pembrolizumab for the treatment of non-small-cell lung cancer. $N$ Engl $J$ Med. 2015;372:2018-28.

13. Gandhi L, Rodriguez-Abreu D, Gadgeel S, Esteban E, Felip E, De Angelis F, et al. Pembrolizumab plus chemotherapy in metastatic non-small-cell lung cancer. N Engl J Med. 2018;378:2078-92.

14. Yang CJ, McSherry F, Mayne NR, Wang X, Berry MF, Tong B, et al. Surgical outcomes after neoadjuvant chemotherapy and ipilimumab for non-small-cell lung cancer. Ann Thorac Surg. 2018;105:924-9.

15. Pisters KM, Vallieres E, Crowley JJ, Franklin WA, Bunn PA Jr, Ginsberg RJ, et al. Surgery with or without preoperative paclitaxel and carboplatin in early-stage non-small-cell lung cancer: Southwest Oncology Group Trial S9900, an intergroup, randomised, phase III trial. J Clin Oncol. 2010;28:1843-9.

16. Felip E, Rosell R, Maestre JA, Rodríguez-Paniagua JM, Morán T, Astudillo J, et al. Preoperative chemotherapy plus surgery versus surgery plus adjuvant chemotherapy versus surgery alone in early-stage non-small-cell lung cancer. J Clin Oncol. 2010;28:3138-45.

17. Gilligan D, Nicolson M, Smith I, Groen H, Dalesio O, Goldstraw P, et al. Preoperative chemotherapy in patients with resectable non-small-cell lung cancer: results of the MRC LU22/NVALT 2/EORTC 08012 multicentre randomised trial and update of systematic review. Lancet. 2007;369:1929-37.

18. Rosell R, Gomez-Codina J, Camps C, Maestre J, Padille J, Cantó A, et al. A randomized trial comparing preoperative chemotherapy plus surgery with surgery alone in patients with non-small-cell lung cancer. $N$ Engl J Med. 1994;330:153-8.

19. Roth JA, Fossella F, Komaki R, Ryan MB, Putnam JB Jr, Lee JS, et al. A randomized trial comparing perioperative chemotherapy and surgery with surgery alone in resectable stage IIIA non-small-cell lung cancer. J Natl Cancer Inst. 1994;86:673-80.

20. Barnett SA, Rusch VW, Zheng J, Park BJ, Rizk NP, Plourde G, et al. Contemporary results of surgical resection of non-small-cell lung cancer after induction therapy: a review of 549 consecutive cases. J Thorac Oncol. 2011;6: 1530-6.

21. Yang CF, Adil SM, Anderson KL, Meyerhoff RR, Turley RS, Hartwig MG, et al. Impact of patient selection and treatment strategies on outcomes after lobectomy for biopsy-proven stage IIIA pN2 non-small-cell lung cancer. Eur J Cardiothorac Surg. 2016;49:1607-13.

22. Kamel MK, Nasar A, Stiles BM, Altorki NK, Port JL. Video-assisted thoracoscopic lobectomy is the preferred approach following induction chemotherapy. J Laparoendosc Adv Tech A. 2017;27:495-500.

23. Petersen RP, Pham D, Toloza EM, Burfeind WR, Harpole DH Jr, Hanish SI, et al. Thoracoscopic lobectomy: a safe and effective strategy for patients receiving induction therapy for non-small-cell lung cancer. Ann Thorac Surg. 2006;82: 214-8; discussion: 219.

24. Krantz SB, Mitzman B, Lufti W, Kuchta K, Wang CH, Howington JA, et al. Neoadjuvant chemoradiation shows no survival advantage to chemotherapy alone in stage IIIA patients. Ann Thorac Surg. 2018;105:1008-16.

25. Doherty MK, Jao K, Shepherd FA, Hazrati LN, Leighl NB. Central nervous system pseudoprogression in a patient treated with PD-1 checkpoint inhibitor. J Thorac Oncol. 2015;10:e100-1.

26. Tanizaki J, Hayashi H, Kimura M, Tanaka K, Takeda M, Shimizu S, et al. Report of two cases of pseudoprogression in patients with non-small-cell lung cancer treated with nivolumab-including histological analysis of one case after tumor regression. Lung Cancer. 2016;102:44-8.

27. Hellmann MD, Rizvi NA, Goldman JW, Gettinger SN, Borghaei H, Brahmer JR, et al. Nivolumab plus ipilimumab as first-line treatment for advanced non-smallcell lung cancer (CheckMate 012): results of an open-label, phase 1, multicohort study. Lancet Oncol. 2017;18:31-41. 
28. Hellmann MD, Ciuleanu TE, Pluzanski A, Lee JS, Otterson GA, AudigierValette C, et al. Nivolumab plus ipilimumab in lung cancer with a high tumor mutation burden. N Engl J Med. 2018;378:2093-104.

Key Words: NSCLC, neoadjuvant, immunotherapy, immune checkpoint inhibition

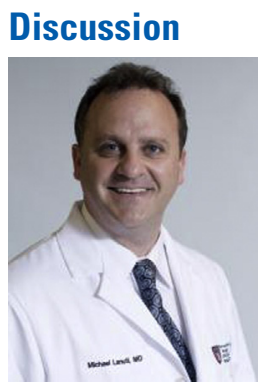

Dr Michael Lanuti (Boston, Mass). Dr Broderick, congratulations to you and your colleagues. There has been a whirlwind of press around this phase I study. The New England Journal published on the nonsurgical details 2 weeks ago. I think this is an important contribution to early stage or resectable lung cancer with the use of induction immunotherapy. The immunotherapy space in advanced non-small cell lung cancer changes almost by the month, and we haven't seen the likes of this activity even with actionable mutations, which often changed every 6 to 12 months. In fact, if you were to construct this trial again, you might have actually constructed it differently based on the most recent data; that is, KEYNOTE 189 published results from 2 weeks ago (pembrolizumab plus chemotherapy for advanced disease), which found improved outcomes regardless of PD-L1 biomarker status. Prior to this particular study, thoracic surgeons might have been more comfortable giving chemotherapy plus immunotherapy (and not immunotherapy alone) routinely for patients harboring stage II and III disease.

Many would agree with the concept of delivering immunotherapy to facilitate priming the immune system with the native tumor in place. Prior to this study, I am not convinced that there was equipoise among surgeons about whether you could effectively treat a stage IIIa patient with just 2 doses of nivolumab and take them to surgery 4 weeks later and think that you might have made a difference.

I was struck by how many patients actually had a near complete response. So almost half of the patients had less than $10 \%$ viable tumor in the resected specimen. I would not have predicted this response with 2 doses of nivolumab.

Those are my comments. I have a few questions for you, Steve. I think this is a great contribution. For the surgeons in the room who have not operated on patients undergoing immunotherapy, it appears that they can have more fibrosis. You reported on a number of conversions from VATS to open (7 cases) and an observation of perihilar fibrosis ... can you give the surgeons a feel of whether these observations were different compared with a stage IIIa patient who might have completed induction chemotherapy or chemotherapy plus radiation?

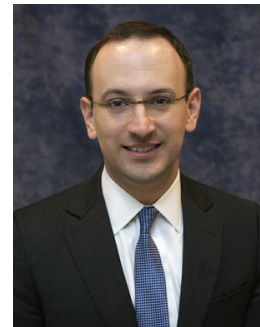

Dr Stephen R. Broderick (Baltimore, $M d)$. It is an incredibly important question and a very, very hard comparison to make, I can tell you that the 2 that I did of nivolumab only and now with Ipi/Nivo, I have done them all open from the get-go. So it's very hard to say if I would have converted had I started VATS.

In my own experience, we primarily offer chemoradiation, and the cases are similar to larger central tumors that receive chemoradiation. I wouldn't say they are any harder or easier than that.

In terms of comparing to neoadjuvant chemotherapy, I don't have a great experience of chemo alone, but even within our own group when we have tried to make those comparisons, we disagree among ourselves; some saying it's harder and some saying it's similar.

Dr Lanuti. Did you compare the major pathologic response across histologies, that is, was there more pathologic response seen in the smokers who might have a higher mutational burden than the nonsmokers?

Dr Broderick. The numbers are very small. We don't see any correlation in this pilot study by histology but we do with tumor mutation burden, and that correlation looks to be significant. Smoking status by the patient's history hasn't panned out, but again, only 20 patients. We expect that that will with higher numbers.

Dr Lanuti. In this clinical trial there were, I believe, 4 patients who had stage I disease. I think what surgeons need to understand is if we are going to delay their therapy by, let's say, 6 weeks ( 2 cycles of immunotherapy followed by a 2 -week cool-off period), are we delaying their curative strategy? So the question would be, how many of the stage I patients had a good pathologic response?

Dr Broderick. I don't have the pathologic response of the stage I's off the top of my head, but it is an important question. So would you offer this to a IA patient? The IA patients are not included in any of the trials we are involved in going forward; it's IB and higher. There were no significant delays to surgery, so most patients start therapy and get to the operating room within 6 to 8 weeks. So if we are potentially offering a therapy that will provide a major pathologic response in many patients, I think that is not a significant delay.

Dr Lanuti. And then the last question is that as we learn about pathologic responders, we look for parameters that would predict that someone would respond. I was disappointed that patients only received a post-treatment CT chest scan; and not a PET scan. Yes, I realize that the tumor FDG avidity might be increased, but one might be able to use a PET scan to discriminate if an immunologic response was present or not in the tumor. I wonder whether it should be considered in future induction immunotherapy studies. 
Dr Broderick. The first handful of patients had a restaging PET that for one reason or another was left out or it wasn't mandated in the protocol. It is in the phase III trial going forward that all patients will have a PET-CT prior to resection so we can hopefully sort that out.

Dr Lanuti. Excellent. Congratulations. Great contribution.

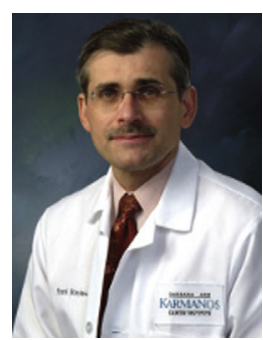

Dr Frank A. Baciewicz, Jr (Detroit, Mich). I enjoyed your paper and I was really interested to see what your experience is, because I have operated now on a few patients after 2 doses of immunotherapy, and the main thing that I have seen have been very plump lymph nodes, which are quite a bit more than the typical ones. I haven't seen any fibrosis that you mentioned. All those patients, of course, got operated within 10 days of their dose, and your mean time I think you said was 18 days out. So I was wondering, does your time of how long waiting maybe change what you have seen? I didn't think this was any worse than chemotherapy or radiation chemotherapy.

The other thing we have noticed is that we seem to have a lot of chest tube output for a lot longer. In fact, I have got somebody back home who is putting out 200 to 300 a day, so I think I'm going to pull their chest tube to make sure their chyle levels are okay. It doesn't look like chyle.

So I just was wondering about your comments about the timing, did that make any difference, and second, on your chest tube output. At least in my experience so far it wasn't that bad.

Dr Broderick. The timing question we just don't know yet. We are talking about only a week or two of difference between what you were doing and what we did in this pilot study. I think as we accrue more patients we might learn more. And the proper interval between therapy and resection is anyone's guess at this moment.

This trial was really designed as a window of opportunity type trial to get our hands on the pathologic specimens and try to figure out how these agents are really working and who they will work best for. So I think we have a long way to go to figure out a lot of the surgical details in terms of patient selection and what is the best way to do these operations.
In terms of the chest tube output, anecdotally, I had a patient who drained a fair amount as well, but the median hospital stay is 4 days. We are not sending a lot of patients home with tubes. We haven't had a lot patients coming back with pleural effusions. So I don't think we are seeing that signal, but again, it's 20 patients.

Dr Baciewicz. You haven't noticed any chyle leaks or anything like that either?

Dr Broderick. We haven't had any chyle leaks. I have seen the large lymph nodes. In fact, the first case I did I probably extended our median operative time while sending a number of frozen sections on in 2 lymph nodes because they looked big and abnormal and I thought they might be involved with cancer, but they were all benign and just big.

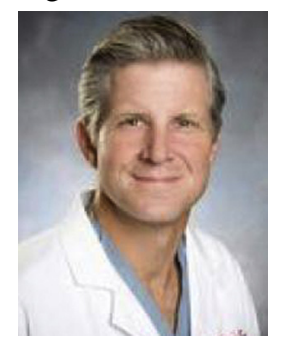

Dr Scott Swanson (Boston, Mass). Great study, well presented. I am struck by this fibrosis. I have done a couple of patients. They have been okay, but certainly not the numbers you have done. It seems to me that it's a little quick to actually get fibrosis. Do you have any more detail on the nature of what that tissue is, have you sent any pathology, do you have any histology, because it seems like it takes longer than a couple of weeks to actually form scar.

The second part is, have you looked back at the radiology to see did anything predict those 6 or 7 cases that were socked in to predict that they were going to be that way?

Thank you.

Dr Broderick. In terms of what is causing the fibrosis, it's hard to say; hopefully it responds to therapy. We see in the tumors a lot of T-cell infiltration and necrosis within the tumor itself. Whether that's happening in nodal disease in the hilum making it more difficult, maybe. What was the second part again?

Dr Swanson. The second was did you look at the 6 or 7 that you couldn't do minimally invasively that were fibrotic? Is there anything predicting that fibrosis?

Dr Broderick. Nothing on the scan that would predict that it would be harder than one that could be done thoracoscopically. 